\title{
Effects of the iron oxide nanoparticle Molday ION Rhodamine $B$ on the viability and regenerative function of neural stem cells: relevance to clinical translation
}

\author{
This article was published in the following Dove Press journal: \\ International Journal of Nanomedicine \\ 27 April 2016 \\ Number of times this article has been viewed
}

\author{
Abhishek Umashankar ${ }^{1,2, *}$ \\ Mandi J Corenblum ${ }^{1, *}$ \\ Sneha Ray $1,2, *$ \\ Michel Valdez ${ }^{3}$ \\ Eriko S Yoshimaru ${ }^{3}$ \\ Theodore P Trouard 3 ,4 \\ Lalitha Madhavan ${ }^{1,4}$ \\ 'Department of Neurology, \\ ${ }^{2}$ Neuroscience and Cognitive \\ Science Undergraduate Program, \\ Undergraduate Biology Research \\ Program, ${ }^{3}$ Department of Biomedical \\ Engineering, ${ }^{4}$ Evelyn F McKnight \\ Brain Institute, University of Arizona, \\ Tucson, AZ, USA \\ *These authors contributed equally \\ to this work
}

\begin{abstract}
An essential component of developing successful neural stem cell (NSC)-based therapies involves the establishment of methodologies to noninvasively monitor grafted NSCs within brain tissues in real time. In this context, ex vivo labeling with ultrasmall superparamagnetic iron oxide (USPIO) particles has been shown to enable efficient tracking of transplanted NSCs via magnetic resonance imaging (MRI). However, whether and how USPIO labeling affects the intrinsic biology of NSCs is not thoroughly understood, and remains an active area of investigation. Here, we perform a comprehensive examination of rat NSC survival and regenerative function upon labeling with the USPIO, Molday ION Rhodamine B (MIRB), which allows for dual magnetic resonance and optical imaging. After optimization of labeling efficiency, two specific doses of MIRB (20 and $50 \mu \mathrm{g} / \mathrm{mL}$ ) were chosen and were followed for the rest of the study. We observed that both MIRB doses supported the robust detection of NSCs, over an extended period of time in vitro and in vivo after transplantation into the striata of host rats, using MRI and post hoc fluorescence imaging. Both in culture and after neural transplantation, the higher $50 \mu \mathrm{g} / \mathrm{mL}$ MIRB dose significantly reduced the survival, proliferation, and differentiation rate of the NSCs. Interestingly, although the lower $20 \mu \mathrm{g} / \mathrm{mL}$ MIRB labeling did not produce overtly negative effects, it increased the proliferation and glial differentiation of the NSCs. Additionally, application of this dose also changed the morphological characteristics of neurons and glia produced after NSC differentiation. Importantly, the transplantation of NSCs labeled with either of the two MIRB doses upregulated the immune response in recipient animals. In particular, in animals receiving the $50 \mu \mathrm{g} / \mathrm{mL}$ MIRB-labeled NSCs, this immune response consisted of an increased number of $\mathrm{CD} 68^{+}$-activated microglia, which appeared to have phagocytosed MIRB particles and cells contributing to an exaggerated MRI signal dropout in the animals. Overall, these results indicate that although USPIO particles, such as MIRB, may have advantageous labeling and magnetic resonance-sensitive features for NSC tracking, a further examination of their effects might be necessary before they can be used in clinical scenarios of cell-based transplantation.
\end{abstract}

Keywords: MRI, neural stem cells, iron oxide nanoparticles, USPIO

\section{Introduction}

The transplantation of neural stem cells (NSCs) has emerged as a promising strategy for the development of cell replacement and neuroprotective therapies with respect to several pathological conditions affecting the nervous system. ${ }^{1-5}$ In fact, clinical trials testing the safety and therapeutic efficacy of NSC transplants in patients with spinal
Department of Neurology, University of Arizona, I50I, North Campbell Avenue, Tucson, AZ 85724, USA

Tel +15206262330

Fax +I 5206262111

Email Imadhavan@email.arizona.edu
International Journal of Nanomedicine 2016:1 | |73|-1748

Dovepress

http://dx,doi.org/10.2147/IN.S102006 (c) (1) (5) 2016 Umashankar et al. This work is published and licensed by Dove Medical Press Limited. The full terms of this license are available at https://www.dovepress.com/terms.php C. hereby accept the Terms. Non-commercial uses of the work are permitted without any further permission from Dove Medical Press Limited, provided the work is properly attributed. For permission for commercial use of this work, please see paragraphs 4.2 and 5 of our Terms (https://www.dovepress.com/terms.php). 
cord injury and amyotrophic lateral sclerosis have recently been initiated (NCT01772810). ${ }^{6,7}$ However, noninvasive technologies that allow dynamic monitoring of transplanted NSCs will be imperative to quantitatively assess the success of such cell-based therapeutic approaches, and improve the design of future clinical trials to support the development of effective NSC therapies.

Iron oxide-based labeling constitutes a simple but effective methodology to label NSCs in vitro, and subsequently track NSC behavior in vivo after neural transplantation. ${ }^{8,9}$ This technique not only allows for the real-time detection of transplanted NSCs through magnetic resonance imaging (MRI) but also supports the later histochemical detection of transplanted cells. In fact, several recent studies have demonstrated the efficient tracking of superparamagnetic iron oxide (SPIO)-labeled NSCs in animal models. ${ }^{10-13}$ Nevertheless, the effects of SPIO labeling on NSC behavior and fate are not fully understood. For eventual clinical use, first, an ideal SPIO agent must not only support the uniform and long-term labeling of NSCs but also exhibit nontoxic qualities such that the intrinsic biology and function of the NSCs (which is of essence in the success of any transplantation approach) is not altered. Second, spurious labeling of neighboring cells (such as microglia and astrocytes) due to SPIO extravasation must be avoided. Such ectopic labeling can lead to the misinterpretation of the MRI signal as surviving transplanted cells.

Here, we comprehensively investigate the influences of the ultrasmall SPIO (USPIO) nanoparticle, Molday ION Rhodamine B (MIRB), on the survival and regenerative capacity of rat NSCs in vitro and in vivo after transplantation into recipient adult Fisher 344 rats. ${ }^{14-16}$ MIRB is conjugated with rhodamine to allow its straightforward detection via fluorescence in histological specimens, while its iron moiety makes it visible in tissue through histochemical methods and MRI. Specifically, after an initial optimization, we analyzed the effects of two doses of MIRB on the morphology, survival, proliferation, and differentiation of both cultured and transplanted NSCs. In addition, we assessed the immune response upon transplantation of the MIRB-labeled NSCs. Our data indicate that although both MIRB doses allow for excellent labeling and extended magnetic resonance (MR)-based tracking of NSCs, they do alter NSC viability and regenerative function.

\section{Materials and methods Overall experimental design}

Primary subventricular zone rat NSCs were treated with specific doses of MIRB, after which they were analyzed over extended periods of time in vitro and in vivo upon neural transplantation. The cultured and grafted NSCs were subjected to MRI scanning, as well as multiple in vitro and in vivo assays to examine NSC survival, regenerative function, as well as the immune response after NSC grafting. Detailed methodologies for all techniques are described in the following sections (Figure 1).

\section{NSC culture}

NSCs were isolated from the subventricular zones of newborn human placental alkaline phosphatase (hPAP) transgenic rat pups using previously described methods. ${ }^{5,17}$ The expression of hPAP allows identification of grafted NPCs, labeled with this human marker, within the brains of wild-type host rats. ${ }^{17,18}$ The NSCs were grown in uncoated dishes in proliferation medium consisting of serum-free Neurobasal-A (Thermo Fisher Scientific, Waltham, MA, USA) supplemented with $20 \mathrm{ng} / \mathrm{mL}$ epidermal growth factor (Cell Sciences, Canton, MA, USA), $10 \mathrm{ng} / \mathrm{mL}$ basic fibroblast growth factor (Cell Sciences), $2 \mu \mathrm{g} /$ $\mathrm{mL}$ heparin (STEMCELL Technologies, Vancouver, BC, Canada), 2\% B27 (Thermo Fisher Scientific), 1\% glutamax (Thermo Fisher Scientific), and 1\% antibiotic-antimycotic (Thermo Fisher Scientific). Cells were maintained under standard conditions at $37^{\circ} \mathrm{C}, 5 \% \mathrm{CO}_{2}$, and $21 \% \mathrm{O}_{2}$. NSCs were passaged every 4-5 days, and all experiments were performed using cells from passages 3 to 7 .

\section{NSC labeling with MIRB}

NSCs were labeled with MIRB, a well-characterized commercially available fluorescent USPIO-based contrast reagent (BioPAL, Inc., Worcester, MA, USA). MIRB can be visualized via both MRI and fluorescence, since it is formed by the reaction of the parent Molday ION SPIO nanoparticle with rhodamine B isothiocyanate. Rhodamine B is a fluorescent dye with an excitation and emission wavelengths of 555 and $565-620 \mathrm{~nm}$, respectively. MIRB is a colloid with an effective diameter of $35 \mathrm{~nm}$, a density of $\sim 1.25 \mathrm{~g} / \mathrm{cm}$, a zeta potential of $\sim+31 \mathrm{mV}$, and $r_{1}$ and $r_{2}$ relaxivity values of 30.4 and $75.8 \mathrm{mM}^{-1} \mathrm{~s}^{-1}$, respectively. It contains an $8 \mathrm{~nm}$ magnetite core, and has dextran coating. After cellular labeling, confocal imaging and transmission electron microscopy have shown that MIRB is found only in endosomes, and does not localize to the nucleus, mitochondria, endoplasmic reticulum, Golgi apparatus, or any other cellular organelle. Also, detailed examination of transmission electron microscopy fields has revealed no traces of electron-dense material corresponding to MIRB on the surface of cells.

Multiple concentrations and times of MIRB treatment $(5,10,20,50,60,80$, and $100 \mu \mathrm{g} \mathrm{Fe} / \mathrm{mL}$ for 18, 20, 30, or 48 hours) were first tested to assess NSC labeling (Figure S1). 


\section{A Overall experimental design}
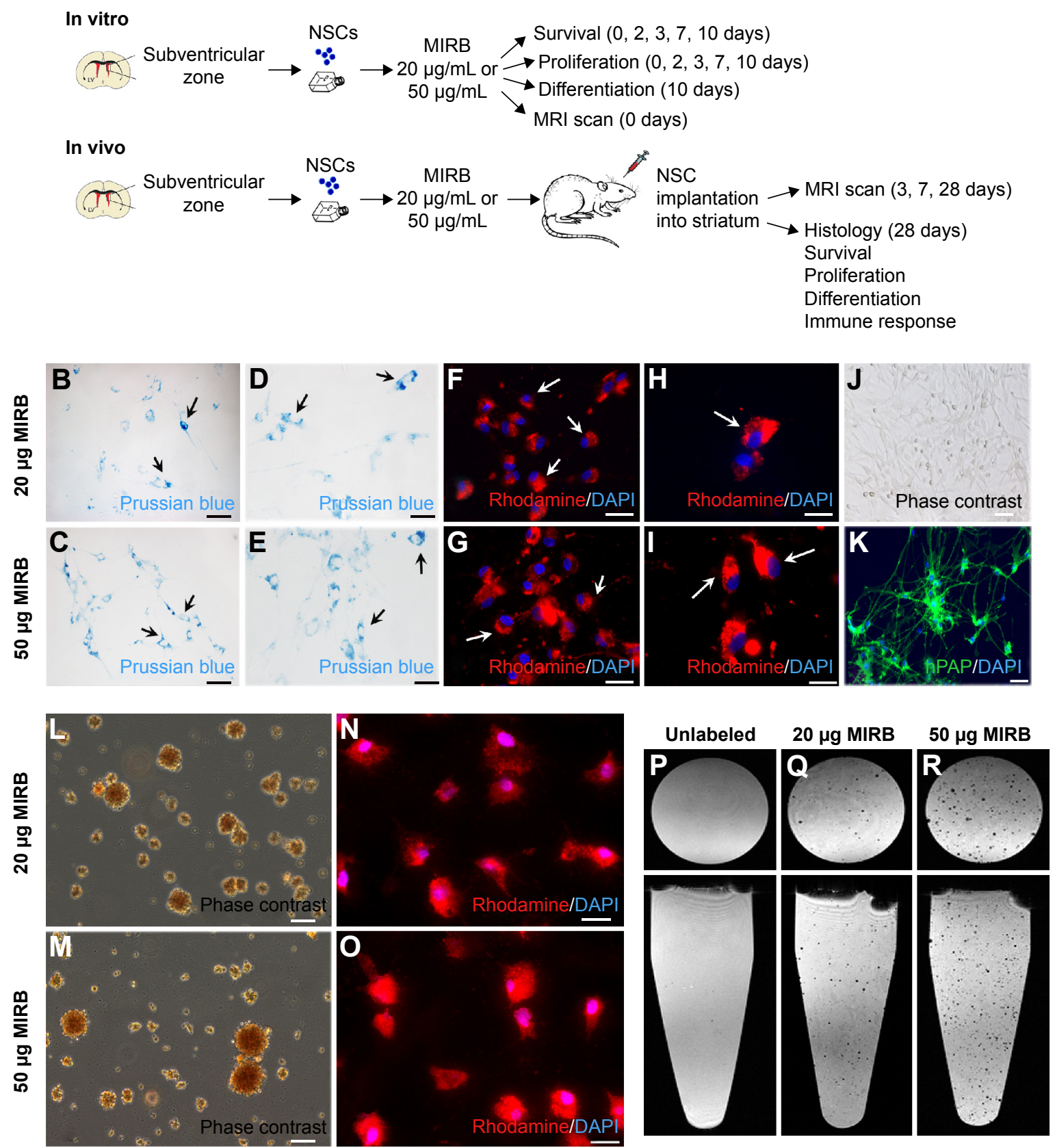

Figure I In vitro MIRB labeling and MRI imaging.

Notes: (A) The overall experimental design of the study. Primary rat NSCs were treated with 20 and $50 \mu g / m L$ doses of MIRB, after which they were assessed directly after staining with Prussian blue (B-E) or under rhodamine fluorescence (F-I). As shown, both doses resulted in excellent incorporation of MIRB within the NSCs (arrows point to examples of labeled cells). Lower magnification images appear in (B), (C), (F), and (G), and higher magnification images appear in (D), (E), (H), and (I). These NSCs (J) had been obtained from newborn rats transgenically expressing hPAP, and therefore also robustly expressed hPAP in culture (K). The MIRB labeling (directly noted as brown staining under phase contrast), at both doses, was strongly maintained in the NSCs after several passages in culture (L and $\mathbf{M}$, at passage I4) and after NSC differentiation for 10 days ( $\mathbf{N}$ and $\mathbf{O}$ ). When tubes containing single-cell suspensions of MIRB-labeled NSCs were scanned via 3D gradient-echo MRI, signal dropout from individual cells was evident in both 20 and $50 \mu \mathrm{g}$ tubes, although not surprisingly, the signal intensity was greater with the $50 \mu \mathrm{g}$ MIRB-labeled cells (P-R). Scale bars: $20 \mu \mathrm{m}$ (B, C, F, and G), $10 \mu \mathrm{m}(\mathbf{D}, \mathbf{E}, \mathbf{H}, \mathbf{I}, \mathbf{N}$, and $\mathbf{O}), 100 \mu \mathrm{m}$ (J and $\mathbf{K})$, and $50 \mu \mathrm{m}$ (L and $\mathbf{M})$.

Abbreviations: MIRB, Molday ION Rhodamine B; MRI, magnetic resonance imaging; NSCs, neural stem cells; DAPI, 4',6-diamidino-2-phenylindole.

These experiments indicated that both 20 and $50 \mu \mathrm{g}$ doses when applied for 20 hours resulted in optimal labeling of the NSCs as visualized by Prussian blue staining and under rhodamine fluorescence. Therefore, for all subsequent experiments, NSCs were trypsinized into a single-cell suspension and treated with 20 or $50 \mu \mathrm{g} / \mathrm{mL}$ MIRB for 20 hours. The cells were subsequently washed in $0.1 \mathrm{M}$ phosphate-buffered saline (PBS), after which they were either processed for various long-term in vitro assays or transplanted into recipient rats for in vivo studies. Details are shown in Figure 1A. 


\section{Measurement of intracellular ROS}

Reactive oxygen species (ROS) measurements were taken using the 2', $7^{\prime}$-dichlorofluorescein diacetate (DCFDA)-Cellular ROS Detection Assay Kit (Abcam, Cambridge, UK). Briefly, 30,000 NSCs were seeded into each well of a 96-well plate (six replicates) and treated with 0,20 , or $50 \mu \mathrm{g} / \mathrm{mL}$ MIRB for 20 hours. Cells were then washed and incubated with $25 \mu \mathrm{M}$ DCFDA at $37^{\circ} \mathrm{C}$ for 45 minutes. Positive controls consisted of NSCs incubated with $50 \mu \mathrm{g}$ tert-butyl hydrogen peroxide solution for 4 hours prior to DCFDA. Negative controls consisted of NSCs incubated without application of DCFDA. Cells were then washed and resuspended in PBS, and analyzed using a Synergy 2 plate reader (BioTek, Winooski, VT, USA) with excitation and emission wavelengths of 485 and $535 \mathrm{~nm}$, respectively. Arbitrary units of fluorescence were calculated by subtracting values from blank wells consisting of PBS only.

\section{In vitro viability assay}

NSC viability was assessed at five time points $(0,2,3,7$, and 10 days) after MIRB labeling using $0.4 \%$ Trypan blue, which is the standard method for assessing cell viability. ${ }^{19}$ Briefly, after the 20-hour incubation with either 20 or $50 \mu \mathrm{g}$ MIRB, NSC cultures were washed and plated at 50,000 cells/well in 24-well plates. Unlabeled NSCs (treated with vehicle/ medium instead of MIRB) acted as a control. At each time point, individual wells of cells were trypsinized, and live cells were counted under a light microscope upon staining with Trypan blue. The assay was done in triplicate using $n=3$ NSC lines gown in parallel.

\section{In vitro proliferation assays}

NSC proliferation was also assessed at 0, 2, 3, 7, and 10 days after MIRB labeling. After the 20-hour incubation with either 20 or $50 \mu \mathrm{g}$ MIRB, NSCs were washed and plated at 50,000 cells/well in 24-well plates, and maintained at $37^{\circ} \mathrm{C}, 5 \% \mathrm{CO}_{2}$, and $21 \% \mathrm{O}_{2}$. Unlabeled NSCs acted as a control. At each time point, individual wells of cells were either trypsinized and counted under a light microscope after staining with $0.4 \%$ Trypan blue (to estimate total number of live cells present compared to time 0 ), or fixed with $4 \%$ paraformaldehyde (PFA) for immunocytochemical analysis using antibodies targeting the cell proliferation marker Ki67 (1:500; Abcam).$^{20}$ Enumeration of Ki67 ${ }^{+}$cells was conducted using a fluorescence microscope. The assays were performed in triplicate using $n=3$ NSC lines grown in parallel.

\section{NSC differentiation}

NSCs were enzymatically dissociated and plated onto polyD-lysine $(0.1 \mathrm{mg} / \mathrm{mL})$ - and laminin $(10 \mu \mathrm{g} / \mathrm{mL})$-coated glass coverslips placed in 24-well plates at a density of 30,000 cells/well in proliferation medium. Subsequently, NSCs were left unlabeled or treated for 20 hours with 20 or $50 \mu \mathrm{g}$ MIRB and washed to remove extraneous MIRB. To induce differentiation, growth factors were retrieved, and the NSCs were maintained in a medium consisting of Neurobasal-A with $1 \%$ glutamax, $2 \%$ B-27, 1\% antibioticantimycotic, and $2 \%$ fetal bovine serum (Atlanta Biologicals, Flowery Branch, GA, USA). Differentiation into neuronal and glial cell types was assessed via immunocytochemistry after 10 days in culture. Five random fields per coverslip were assessed, and the percentage of cells (stained with the nuclear marker 4',6-diamidino-2-phenylindole [DAPI]) expressing Tuj1 (for neurons), S100 $\beta$ (for astrocytes), or RIP (for oligodendrocytes) was counted under a $\times 20$ lens. NSC lines $(n=3)$ were grown in parallel and assessed in triplicate.

\section{Immunocytochemistry}

MIRB-labeled or unlabeled NSCs plated on poly-D-lysineand laminin-coated glass coverslips were immunostained following published protocols. ${ }^{4,21}$ Cells were briefly washed and then blocked with $1 \%$ bovine serum albumin (SigmaAldrich, St Louis, MO, USA) in 1× PBS (Thermo Fisher Scientific) containing $0.4 \%$ triton X-100 (Sigma-Aldrich) and $2 \%$ normal goat serum (Thermo Fisher Scientific). After incubation overnight at $4{ }^{\circ} \mathrm{C}$ with primary antibodies, cells were treated with appropriate secondary antibodies (1:500) coupled to fluorochromes Alexa 488, 594, or 647 (Thermo Fisher Scientific = Molecular Probes, Eugene, OR, USA) and counterstained with DAPI (Thermo Fisher Scientific). Primary or secondary antibodies were deleted under control conditions. The primary antibodies used were as follows: hPAP (1:500; Sigma-Aldrich), Nestin (1:300; EMD Millipore, Billerica, MA, USA), Tuj1 (1:300; Covance, Princeton, NJ, USA), GFAP (1:1,000; EMD Millipore), RIP (1:500; EMD Millipore), and Ki67 (1:500; Abcam).

\section{In vitro MRI}

NSCs were trypsinized and treated with 20,50 $\mu \mathrm{g}$ MIRB, or left unlabeled (control) for 20 hours. Cells were then washed and resuspended at 200,000 cells $/ \mathrm{mL}$ before fixation with $4 \%$ PFA. Half of the cell suspension $(100,000$ cells $/ 0.5 \mathrm{~mL})$ was mixed with $0.5 \mathrm{~mL}$ of $1 \%$ agarose solution containing $1 \mathrm{mM}$ gadolinium-based contrast agent (MultiHance; Bracco Diagnostics Inc., Milan, Italy) and allowed to solidify. MRI was carried out on a $7 \mathrm{~T}$ Bruker BioSpec (Bruker Corporation, Billerica, MA, USA) with gradients capable of $430 \mathrm{mT} / \mathrm{m}$ along the $x$-, $y$-, and $z$-axis. A $72 \mathrm{~mm}$ linear volume coil was used for excitation, and a four-channel phased array surface 
coil was used for reception. Tubes containing MIRB-loaded and -unloaded stem cells were imaged simultaneously at room temperature using a $3 \mathrm{D}$ gradient-echo sequence (repetition time $=100 \mathrm{~ms}$, time to echo $=10 \mathrm{~ms}, 40^{\circ}$ flip angle, $3.60 \times 1.92 \times 1.00 \mathrm{~cm}$ field of view, $100 \mu \mathrm{m}$ isotropic resolution, 32-minute scan time).

\section{Prussian blue staining}

Prussian blue cell staining reagent (BioPAL, Inc.) is formulated for use with MIRB and other iron-based nanoparticles in order to visualize the presence of iron deposits found inside cells. After fixing cells and tissue with 4\% PFA, a working solution of Prussian blue reagent was prepared according to the manufacturer's instructions. A sufficient amount of working solution was applied to cover cells or tissue sections and incubated at room temperature for 15 minutes. Cells and tissue were washed with $0.1 \mathrm{M}$ PBS and visualized via light microscopy.

\section{Animals}

Animals were housed and treated according to the rules and regulations of the National Institutes of Health and Institutional Guidelines on the Care and Use of Animals. The University of Arizona's Institutional Animal Care and Use Committee approved all experimental procedures. Experimental animals, namely male Fisher 344 rats (250-300 g; Harlan Laboratories, Indianapolis, IN, USA), and the hPAP transgenic rat colony (from which the NSCs were isolated) were maintained at the University of Arizona animal care facility. All animals were kept on a standard 12-hour lightdark cycle with availability of food and water ad libitum.

\section{Experimental paradigm}

NSCs were transplanted into the striatum of naïve Fisher 344 rats, and sacrificed at 1 month post-transplantation. All experimental rats were anesthetized with a cocktail of $44 \mathrm{mg} / \mathrm{kg}$ ketamine and $10 \mathrm{mg} / \mathrm{kg}$ xylazine before being placed on a stereotaxic device. Animals were maintained under $2 \%$ isoflurane for the duration of surgical procedure.

\section{NSC transplants}

One lakh undifferentiated NSCs $(50,000$ cells/ $\mu$ L of Hank's balanced salt solution, $2 \mu \mathrm{L} /$ site) were stereotaxically infused unilaterally into the left striatum (anteroposterior +0.2 , mediolateral +3.0 , dorsoventral -5.5 from bregma), at a rate of $0.5 \mu \mathrm{L} / \mathrm{min}$, as described previously. ${ }^{17}$

The number of animals in each experimental group was as follows: sham (only buffer), $n=5$; unlabeled NSCs, $n=7$; $50 \mu \mathrm{g}$ MIRB-labeled NSCs, n=7; and $20 \mu \mathrm{g}$ MIRB-labeled NSCs, $\mathrm{n}=7$.
At 1 month post-NSC transplantation, animals were sacrificed using pentobarbital (60 mg/kg), perfused with $4 \%$ PFA, and their brains were extracted. The brains were subsequently post-fixed in $4 \%$ PFA, sunk through a $30 \%$ sucrose solution, and sectioned coronally at $40 \mu \mathrm{m}$ thickness on a freezing sliding microtome for histological studies.

\section{In vivo $M R I$}

MRI was carried out on the same MRI system as the in vitro imaging (described in the "In vitro MRI" section). Rat brain imaging was performed using parameters similar to the in vitro scanning, namely using a 3D gradient-echo sequence (repetition time $=100 \mathrm{~ms}$, time to echo $=10 \mathrm{~ms}, 40^{\circ}$ flip angle, $3.84 \times 2.56 \times 1.92 \mathrm{~cm}$ field of view, $150 \mu \mathrm{m}$ isotropic resolution, 82-minute scan time). Rats were anesthetized using oxygen gas with $2.5 \%$ isoflurane and were secured on a heated animal cradle equipped with a bite bar and ear bars. Core temperature and respiration were monitored during imaging.

\section{Immunohistochemistry}

Immunohistochemistry was performed according to standard protocols. ${ }^{4,21}$ Sections were blocked (10\% normal goat serum, $0.5 \%$ triton $\mathrm{X}-100$ in tris-buffered saline $[\mathrm{pH} 7.4]$ ) and incubated in primary antibody overnight at room temperature. Primary antibodies were detected in a 2-hour incubation at room temperature with secondary antibodies (1:250) coupled to fluorochromes Alexa 488, 594, 647 (Thermo Fisher Scientific $=$ Molecular Probes) and counterstained with DAPI (Thermo Fisher Scientific). Primary antibodies utilized were as follows: hPAP (1:500; Sigma-Aldrich), Ki67 (1:500; Abcam), Tuj1 (1:100; Covance), GFAP (1:500 Clone-GA5: 1:500; EMD Millipore), and RIP (1:200; EMD Millipore). Control conditions constituted the deletion of the primary antibody or secondary antibody and the inclusion of relevant isotype-specific antibodies in sera instead of the omitted antibodies.

\section{In vivo quantification}

To estimate graft proliferation, $\mathrm{hPAP}^{+}$-grafted NSCs expressing Ki67 were counted using confocal microscopy in three adjacent sections/animal under a $\times 63$ lens. Data were expressed as mean \pm standard error of the mean (SEM) of percentage of $\mathrm{Ki} 67^{+}$cells per total $\mathrm{hPAP}^{+}$cells per animal. Differentiation of the grafted $\mathrm{hPAP}^{+}$NSCs into Tuj $1^{+}$ (neurons), $\mathrm{GFAP}^{+}$(astrocytes), $\mathrm{RIP}^{+}$(oligodendrocytes) was also assessed via confocal microscopy. Three adjacent sections were examined under $\mathrm{a} \times 40$ lens, and the co-localization rate of Tuj1, GFAP, or RIP with hPAP was calculated via the Leica LAS software version 2.7.3.9723 (Leica Microsystems, 
Wetzlar, Germany). Data were expressed as mean \pm SEM of percent co-localization rate, between hPAP and each of the differentiation markers, per animal. To quantify the immune response, the mean area of the striatum which contained $\mathrm{CD} 68^{+}$microglia, and the density of $\mathrm{CD} 68^{+}$microglia within this area, was estimated on $\times 40$ confocal images using SimplePCI software version 6.5.1 (Hamamatsu Corporation, Hamamatsu, Japan). Briefly, after optimal thresholding of the image, regions of interest (ROIs) were drawn, and the area and density of objects (CD68 ${ }^{+}$cells) were automatically calculated through SimplePCI software. Data were expressed as mean \pm SEM of mean ROI area or the fraction of ROI area where $\mathrm{CD}^{+} 8^{+}$cell bodies were present (as a measure of density). To estimate graft size, graft areas (in $\mu \mathrm{m}^{2}$ ) were estimated via the SimplePCI software after drawing ROIs around the graft periphery. All the quantification occurred in three to four animals per experimental group.

\section{Microscopy}

A Zeiss AxioImager A1 (Zeiss, Jena, Germany) inverted microscope with phase capability, with an AxioCam MRc camera and associated AxioVision software (version: Axio Vision Rel.4.8.2; Zeiss), was used to qualitatively analyze the NSCs in culture. Fluorescence analysis on brain sections was performed using a Leica SP5-II confocal microscope (Leica Microsystems). $Z$ sectioning was performed at $1-2 \mu \mathrm{m}$ intervals in order to verify the co-localization of markers. Image extraction and analysis was conducted via the Leica LAS software. In vitro fluorescence analysis was conducted using a Zeiss M2 Imager microscope connected to an AxioCam MRm digital camera.

\section{Statistical analyses}

GraphPad Prism 6 software was used for statistical analyses. For comparing two groups, $t$-tests were used. For comparisons between three or more groups, analysis of variance (ANOVA) followed by Tukey's or Bonferroni's post hoc test for multiple comparisons between treatment groups was conducted. Differences were accepted as significant at $P<0.05$. Additional statistical details pertaining to each experiment are provided within the relevant results and legend sections.

\section{Results}

\section{Labeling efficiency and MR sensitivity of MIRB in NSCs}

Our previous studies have extensively characterized the properties and therapeutic potential of rat NSCs expressing hPAP, a human marker which allows for the identification of the NSCs in vivo after transplantation into recipient animals. ${ }^{4,5,17}$
Using these well-characterized cells (Figure 1), we tested several doses of MIRB to determine the optimal concentration and time required for the clear and uniform labeling of NSCs in culture (Figure S1). MIRB is a commercially available iron oxide nanoparticle, which has been shown to robustly label and support MRI-based tracking of a variety of stem cells..$^{10,14,21}$ In addition to enabling the detection of labeled cells via MRI and post hoc Prussian blue staining, MIRB also offers the additional advantage of easy and direct visualization of the cells via emission of red fluorescence due to its conjugation with rhodamine. As shown, a 20-hour treatment with 20 or $50 \mu \mathrm{g} / \mathrm{mL}$ MIRB resulted in robust NSC labeling as detected by Prussian blue (Figure 1B-E) and rhodamine expression (Figure 1F-I). In addition, excellent MIRB labeling (noted even under phase contrast as brown coloration of cells) was maintained in the NSCs even after several passages in culture (Figure 1L and $\mathrm{M}$ ) and also after the cells were induced to undergo differentiation for 10 days (Figure $1 \mathrm{~N}$ and $\mathrm{O}$ ). We also imaged single-cell suspensions of 20 and $50 \mu \mathrm{g}$ MIRB-treated NSCs with MRI. Here, individual cells were detectable in vials containing $20 \mu \mathrm{g}$ as well as $50 \mu \mathrm{g}$ MIRB-treated NSCs. However, the contrast signal in tubes containing $50 \mu \mathrm{g}$ MIRB-treated NSCs was more evident than those containing $20 \mu \mathrm{g}$, as depicted in the axial and sagittal images in Figure 1P-R.

\section{Effects of MIRB on the in vitro viability and proliferation of NSCs}

The viability and proliferative capacity of the 20 and $50 \mu \mathrm{g}$ MIRB-labeled NSCs were assessed at several time points, after the cessation of MIRB labeling, across a period of 10 days (Figure 2). As shown in Figure 2A-F, there was a clear loss of cells observed in $50 \mu \mathrm{g}$ MIRB cultures at 10 days (F) compared to $20 \mu \mathrm{g}$ (E) and control (unlabeled, D) NSCs. Measurements of intracellular ROS soon after the 20-hour MIRB treatment showed that increased ROS were present in NSCs treated with both doses, although at a significantly higher level in the $50 \mu \mathrm{g} / \mathrm{mL}$-labeled NSCs (Figure 2J). Trypan blue staining at 0, 2, 3, 7, and 10 days in culture determined that the percentage of live cells was not altered in $20 \mu \mathrm{g}$ MIRB cultures in comparison to control cells (Figure 2K). However, the $50 \mu \mathrm{g}$ MIRB cultures showed a significant loss of live cells at 0 and 2 days, after which the viability improved to reach control levels (Figure 2L). In addition, the total number of cells/well in $20 \mu \mathrm{g}$ MIRB cultures was comparable to control cultures at every time point (Figure 2M), whereas this measure was significantly lower at all time points in the $50 \mu \mathrm{g}$ NSC cultures (Figure $2 \mathrm{~N}$ ). These data indicated that the viability and 

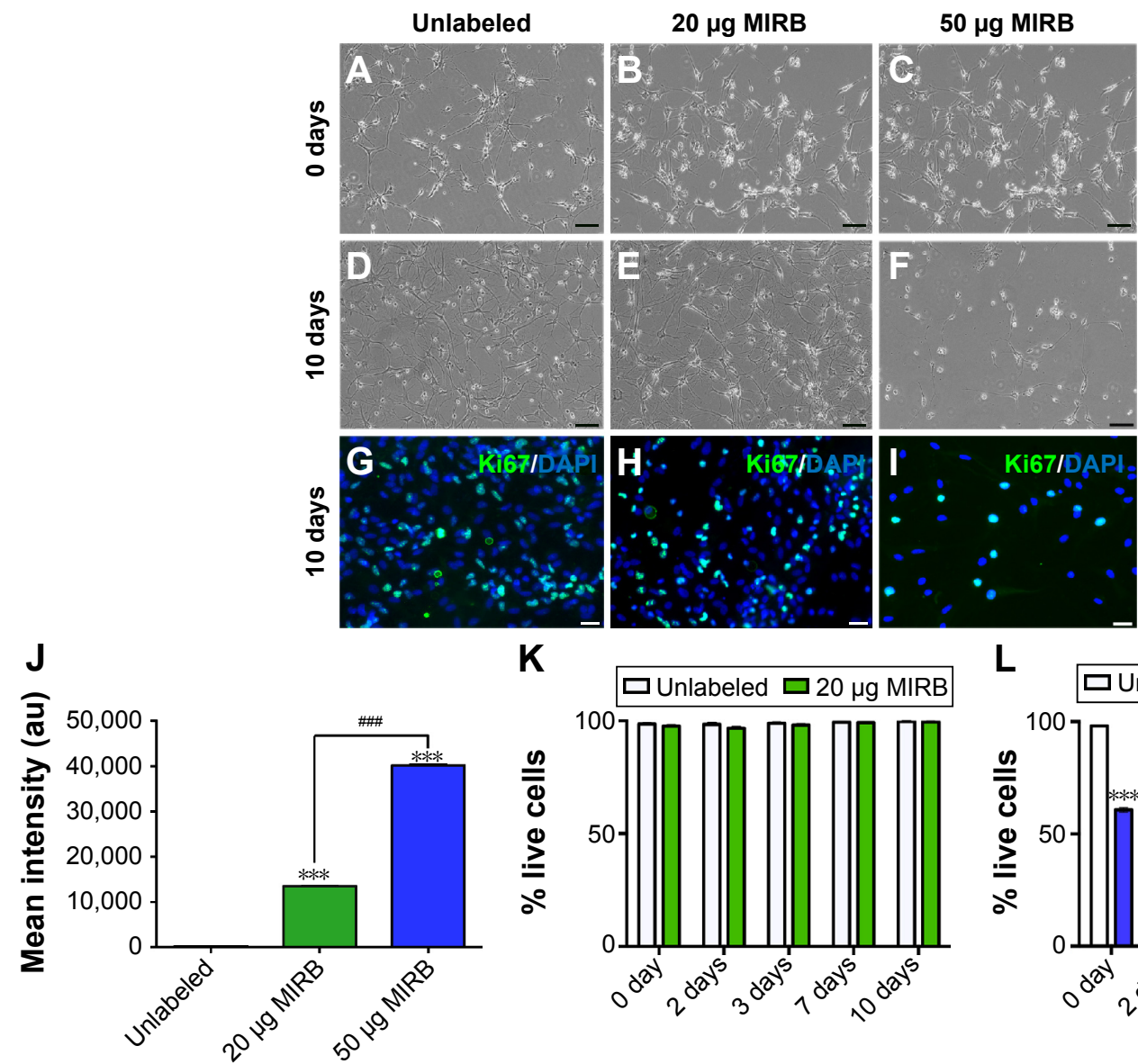

K

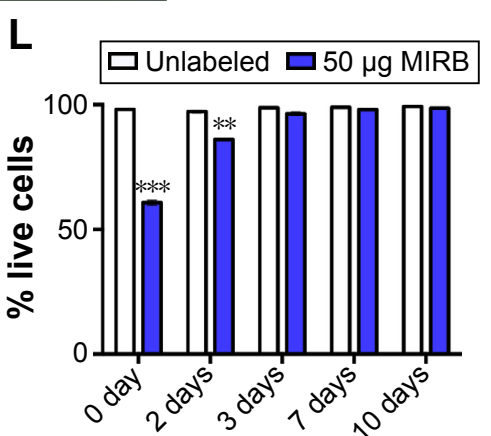

M

$\mathbf{N}$

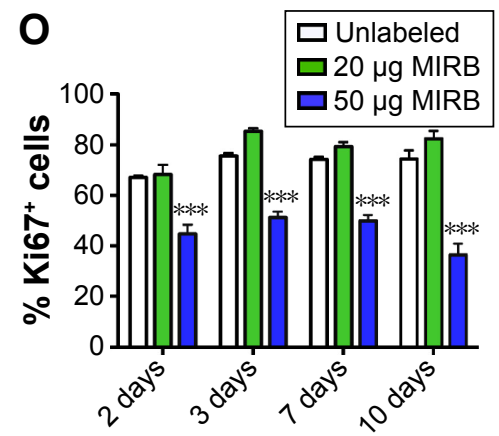

Figure 2 MIRB labeling affects NSC viability and proliferation.

Notes: The viability of $50 \mu \mathrm{g}$ MIRB-labeled NSCs was assessed across a 10-day culture period. As shown, a significant cell loss was noted in the $50 \mu \mathrm{g}$ cultures at 10 days ( $\mathbf{C}$ and $\mathbf{F}$ ), as compared with control (unlabeled; $\mathbf{A}$ and $\mathbf{D}$ ) and $20 \mu$ g-labeled cells (B and E). Measurement of intracellular ROS at the end of the 20-hour MIRB-labeling period (via a DCFDA assay) indicated increased levels within NSCs treated with both MIRB doses, although the level was found to be significantly higher in the $50 \mu g$ MIRBlabeled cells $(\mathrm{J})$. When the percentage of live cells was calculated via Trypan blue staining, no changes were seen in the $20 \mu \mathrm{g}$ cultures (K). However, the $50 \mu \mathrm{g}$ cultures showed a significant reduction in the live cell numbers at baseline and 2 days in culture, after which they maintained $\sim 100 \%$ viability at 3, 7, and 10 days (L). Similarly, the total number of cells/well of $20 \mu \mathrm{g}$ MIRB-labeled NSCs was comparable to control cultures (M), but wells with $50 \mu \mathrm{g}$ MIRB NSCs showed fewer cells at all time points (N). In addition, immunocytochemical analysis using a Ki67 antibody indicated a lower number of proliferating cells in the $50 \mu \mathrm{g}$ cultures compared to control and $20 \mu \mathrm{g}$ cultures (G-I). Quantification of Ki67+ cells confirmed that there were indeed significantly fewer Ki67 cells in the $50 \mu g$ cultures and some increase (although not significant) noted

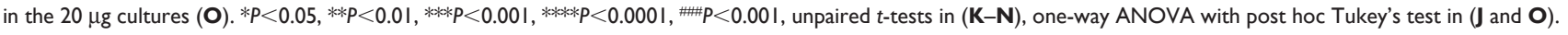
Scale bars: $30 \mu \mathrm{m}(\mathbf{A}-\mathbf{F})$ and $10 \mu \mathrm{m}(\mathbf{G}-\mathbf{I})$.

Abbreviations: MIRB,Molday ION Rhodamine B;NSC, neural stem cell; ROS, reactive oxygen species;DAPI,4',6-diamidino-2-phenylindole;DCFDA, 2',7'-dichlorofluorescein diacetate;ANOVA, analysis of variance.

proliferative rate of NSC cultures treated with $50 \mu \mathrm{g}$ MIRB had been significantly compromised. To further assess the proliferative capacity of the MIRB-labeled cells, the expression of Ki67, an antigen expressed by proliferating cells during all active phases of the cell cycle, was examined in the NSCs via immunocytochemistry (Figure 2G-I). It was observed that NSCs labeled with $50 \mu \mathrm{g}$ MIRB contained substantially lower numbers of Ki67-expressing cells, at every time point examined, compared to control and $20 \mu \mathrm{g}$ MIRB-labeled NSCs (Figure 2O). Interestingly, the $20 \mu \mathrm{g}$ 
MIRB cultures showed higher percentages of Ki67-labeled cells (although not significant) at most of the examined time points (Figure 2O).

\section{MIRB alters the morphology and lineage differentiation of the NSCs}

Next, we differentiated the control and MIRB-labeled NSCs to assess their multipotentiality (capacity to generate all three major neural lineages, namely neurons, astrocytes, and oligodendrocytes) and resultant morphology (Figure 3). It was noted that both 20 and $50 \mu \mathrm{g}$ MIRB NSCs differed in shape and size from control cells, under light microscopy, after the 10-day differentiation period (Figure 3A-C). On immunohistochemical examination, we found that all groups of NSCs robustly expressed the NSC antigen Nestin at baseline (Figure 3D-F) but showed alterations in their ability to generate neurons and glia after differentiation. More specifically, the $20 \mu \mathrm{g}$ MIRB-labeled NSCs had produced similar percentages of Tuj $1^{+}$neurons, $\mathrm{GFAP}^{+}$astrocytes, and $\mathrm{RIP}^{+}$oligodendrocytes as their control unlabeled counterparts when examined after 10 days of differentiation (Figure 3P). However, the $50 \mu \mathrm{g}$ MIRB-labeled NSCs showed substantial reductions in neuronal and glial cell numbers (Figure 3Q). Furthermore, the morphological characteristics of the neurons, astrocytes, and oligodendrocytes appeared particularly altered in the $50 \mu \mathrm{g}$ NSC cultures. Specifically, although the expression of the neuronal and glial antigens was seen in some $50 \mu \mathrm{g}$ MIRB-labeled NSCs, the cells were stunted in growth, and the typical mature morphology of neurons and glial cells (with multiple processes; Figure 3G, J, and $\mathrm{M}$ ) was not seen (Figure 3I, L, and O). In addition, even at baseline (before differentiation), the Nestin-expressing NSCs already showed changes in cytoskeletal architecture in the $50 \mu \mathrm{g}$ MIRB group (Figure 3D-F). Interestingly, even though the $20 \mu \mathrm{g}$ MIRB NSCs did not show changes in terms of the numbers of neurons and glia generated, the morphology of differentiated cells was different from those in control NSC cultures (Figure 3H, K, and N). Neurons in the $20 \mu \mathrm{g}$ MIRB cultures predominantly exhibited a more differentiated phenotype with larger cell bodies and longer axonal processes, and astrocytes displayed a fibrous morphology (vs protoplasmic features predominantly seen in the control culture astrocytes) (Figure 3J and K). Oligodendrocytes also displayed exaggerated differentiation in the $20 \mu \mathrm{g}$ MIRB cultures (Figure $3 \mathrm{~N}$ ), and showed greater ramification of processes compared to control oligodendrocytes which appeared comparatively immature (Figure 3M).

\section{Detection and assessment of viability and migration of transplanted MIRB-labeled NSCs via extended MRI-based in vivo tracking and post hoc histology}

We tested whether the MIRB-labeled NSCs could be followed effectively in vivo through MRI after their transplantation into rat brains (Figure 4). More specifically, unlabeled NSCs or NSCs labeled with either 20 or $50 \mu \mathrm{g} / \mathrm{mL}$ MIRB were transplanted unilaterally into the striata of naïve adult rats. The animals were then imaged using a 3D gradient-echo MRI at 3, 7, and 28 days after NSC transplantation. As shown in Figure 4A-C, in animals injected with NSCs labeled with $50 \mu \mathrm{g}$ MIRB, a large, circular signal dropout (arrows) at the injection site was clearly visible at all time points studied, signifying the presence of iron-loaded cells. Animals injected with NSCs labeled with $20 \mu \mathrm{g}$ MIRB showed a persistent, but much smaller, circular signal dropout (Figure 4D-F, arrows). Control animals injected with unlabeled NSCs showed signal dropouts in the injection site that had a more branched architecture, and that slightly decreased over time (Figure 4G-I). MRI images were also obtained from sham animals, injected only with buffer, and showed persistent signal dropout in the needle track region similar to previous reports (Figure S2) by Williams. ${ }^{22}$

Subsequent histological analysis at 1 month revealed the presence of $\mathrm{hPAP}^{+}$-grafted cells in corresponding areas of the striatum where the MRI signal dropout had been noted in animals receiving 20 and $50 \mu \mathrm{g}$ MIRB-labeled NSCs (Figure 4N and $\mathrm{O}$ ). The MIRB-labeled grafted NSCs were also identifiable by Prussian blue staining (Figure 4J and K) and rhodamine fluorescence (Figure $4 \mathrm{~L}$ and $\mathrm{M}$ ). These data indicated that the MIRB-labeled cells could be effectively tracked over an extended period via real-time in vivo MRI, and post hoc histological analysis. The MRI and histological data also indicated that the grafted NSCs had not migrated extensively, and had stayed confined to the area of transplantation upon grafting. Moreover, contrary to the MRI data, quantitative analysis of graft sizes in the NSC-transplanted animals showed that on average, the area of $50 \mu \mathrm{g}$ MIRB grafts was significantly smaller $\left(71,310.5 \pm 12,355 \mu^{2}\right)$ than control $\left(134,693.3 \pm 10,217.4 \mu \mathrm{m}^{2}\right)$ or $20 \mu \mathrm{g}\left(152,826.7 \pm 14,235 \mu \mathrm{m}^{2}\right)$ MIRB-labeled grafts $(P<0.01$, one-way ANOVA). These data suggested that the higher dose of MIRB substantially reduced the viability of the transplanted NSCs. 

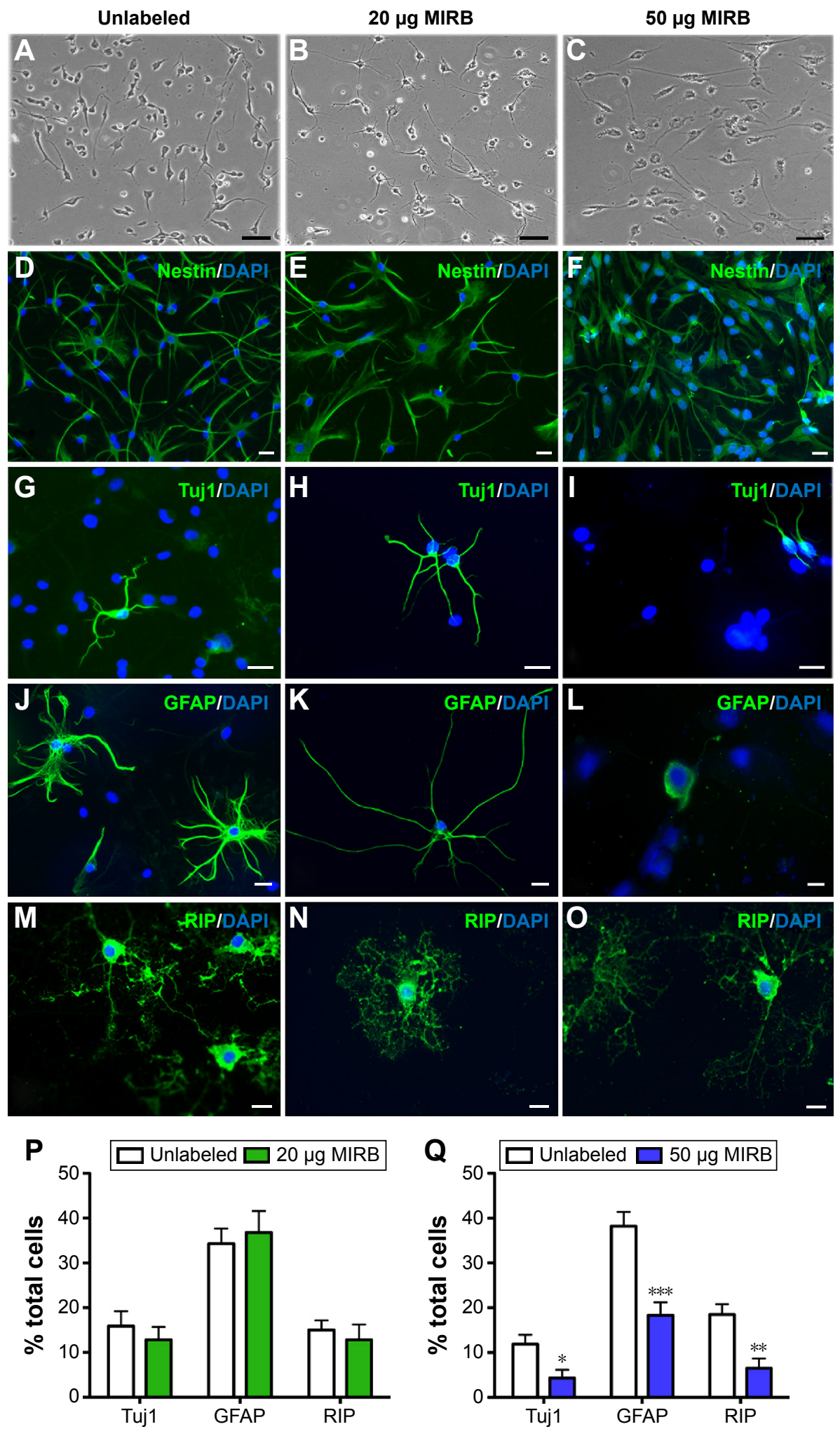

Figure 3 NSC differentiation rate and morphology are altered by MIRB labeling.

Notes: The morphology of control, 20, and $50 \mu \mathrm{g}$ MIRB NSCs differed after a I0-day differentiation period (A-C). Immunocytochemical analysis revealed that at baseline, all NSC groups robustly expressed the immature NSC marker Nestin, yet they showed differences in cytoskeletal structure (D-F). Upon differentiation, the number of Tuj ${ }^{+}$ neurons, GFAP ${ }^{+}$astrocytes, and RIP+ oligodendrocytes was significantly reduced in the $50 \mu \mathrm{g}$ cultures $(\mathbf{Q})$, with an absence of classic neuronal and glial morphology noted (I, L, and $\mathbf{O}$ ), when compared to controls (G, J, and $\mathbf{M})$. In the $20 \mu \mathrm{g}$ cultures, there were no changes in numbers of neurons and glia generated (P), but the morphology of cells was altered $(\mathbf{H}, \mathbf{K}$, and $\mathbf{N})$. More specifically, more mature and well-ramified neuronal and oligodendrocyte cells were observed, and astrocytes showed a fibrous (vs protoplasmic) feature ( $\mathbf{N}) . * P<0.05$, $* * P<0.0$ I, $* * * P<0.00$ I, one-way ANOVA with post hoc Tukey's test. Scale bars: $30 \mu \mathrm{m}(\mathbf{A}-\mathbf{C})$ and I0 $\mu \mathrm{m}$ (D-O). Abbreviations: NSC, neural stem cell; MIRB, Molday ION Rhodamine B; ANOVA, analysis of variance; DAPI, 4',6-diamidino-2-phenylindole. 

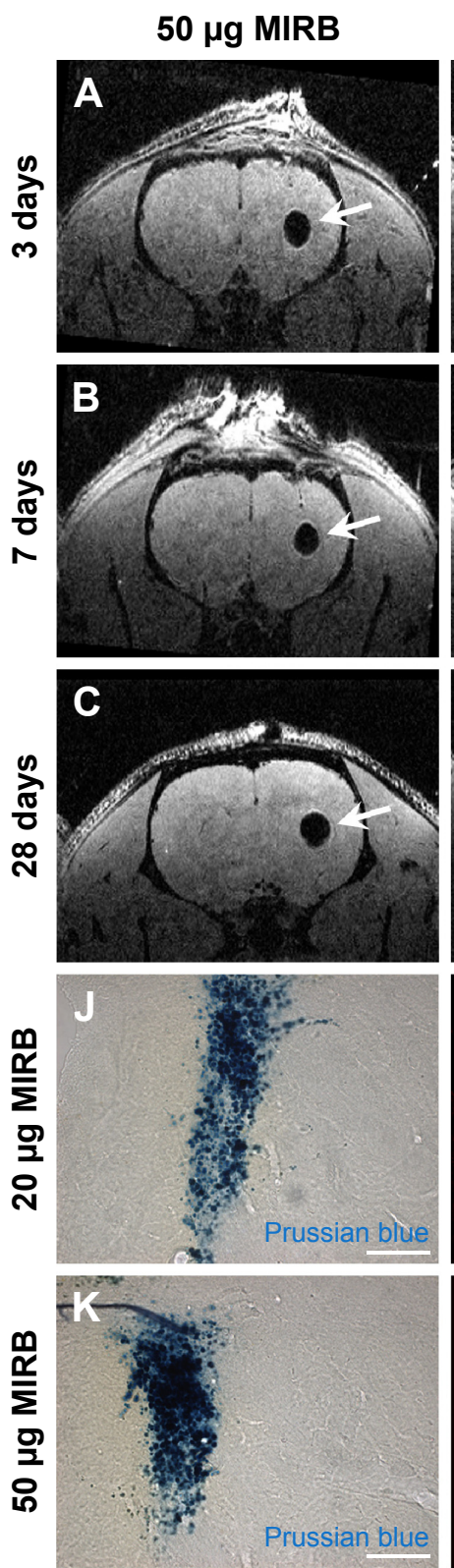
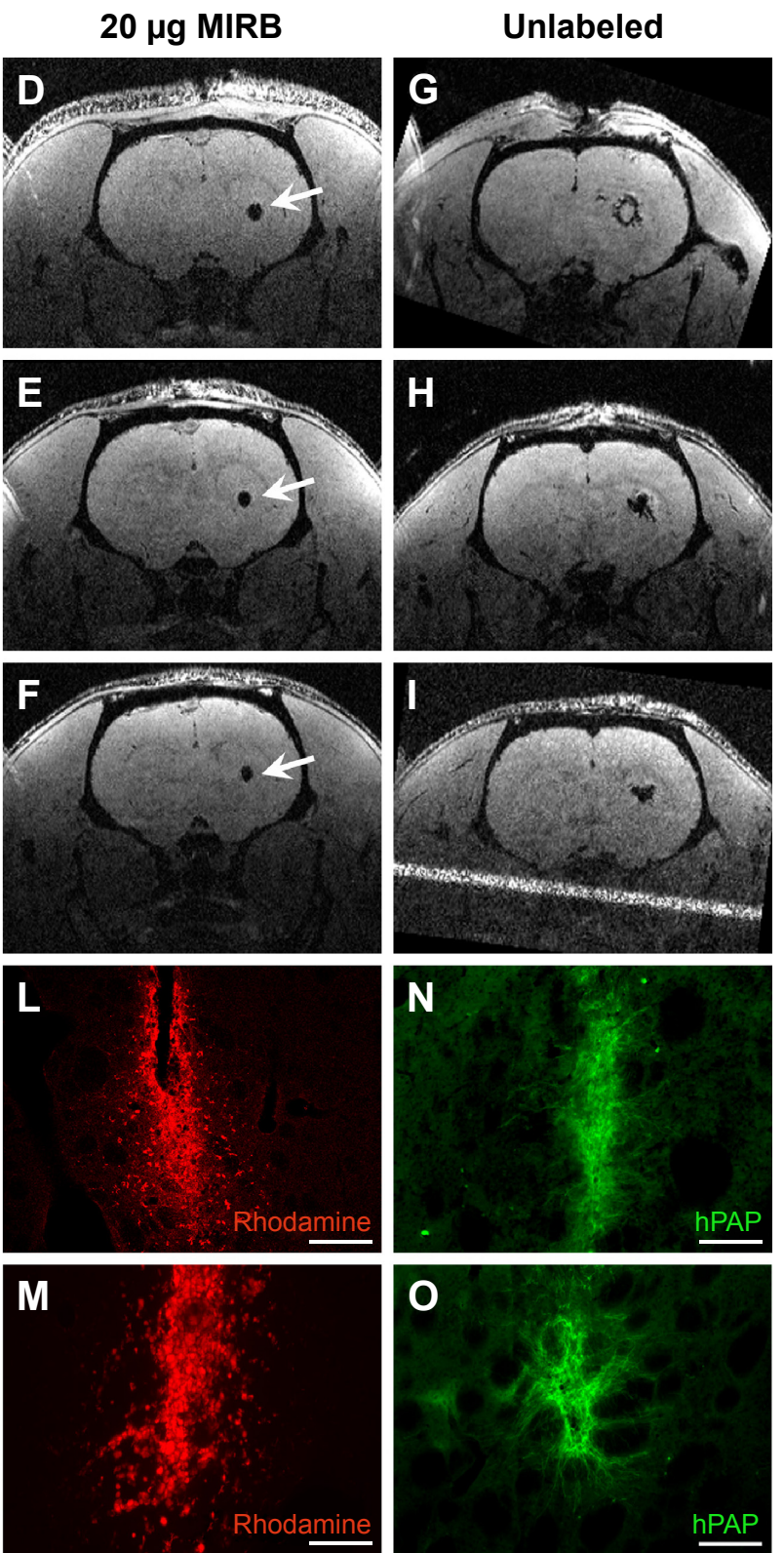

Figure 4 Extended in vivo tracking of MIRB-labeled NSCs.

Notes: We transplanted control (unlabeled), $20 \mu \mathrm{g}$ or $50 \mu \mathrm{g}$ MIRB-labeled NSCs into the striata of adult rats, and imaged the animals longitudinally over a period of I month. Distinct and typical signal dropout (arrows, indicating iron oxide-labeled cells) in the striatum was noted at 3, 7, and 28 days post-transplantation in animals receiving the $20 \mu \mathrm{g}$ or $50 \mu \mathrm{g}$ MIRB-labeled NSCs but not unlabeled cells (A-I). Also, the observed signal dropouts in the $50 \mu \mathrm{g}$ animals were significantly stronger than in $20 \mu \mathrm{g}$ animals. Upon later histological examination, areas corresponding to the regions where the signal dropout were noted in $20 \mu \mathrm{g}$ and $50 \mu \mathrm{g}$ MIRB animal groups showed the clear presence of hPAP+-grafted NSCs ( $\mathbf{N}$ and $\mathbf{O})$ which could be visualized via rhodamine fluorescence $(\mathbf{L}$ and $\mathbf{M})$ as well as Prussian blue staining $(\mathbf{J}$ and $\mathbf{K})$. Scale bars: $200 \mu \mathrm{m}$. Abbreviations: MIRB, Molday ION Rhodamine B; NSC, neural stem cell.

\section{MIRB compromises the proliferation and} differentiation ability of transplanted NSCs in vivo

Proliferation in the striatal NSC grafts was examined through immunohistochemical staining using antibodies targeting the cell cycle antigen $\mathrm{Ki67}$, at 1 month post-transplantation (Figure 5, higher magnification image in Figure Dd).
Quantitative analysis determined that on average, the percentage of Ki67-expressing cells within unlabeled control grafts was negligible (1.0 \pm 0.5 ; Figure $5 \mathrm{Q})$. On the other hand, although the percentage of Ki67 cells was similar in the $50 \mu \mathrm{g}$ MIRB-labeled grafts (1.2 \pm 0.8$)$, it was significantly increased in $20 \mu \mathrm{g}$ MIRB-labeled grafts $(P<0.05 ; 7.1 \pm 1.3)$. When the differentiation potential of the grafted NSCs was analyzed by 

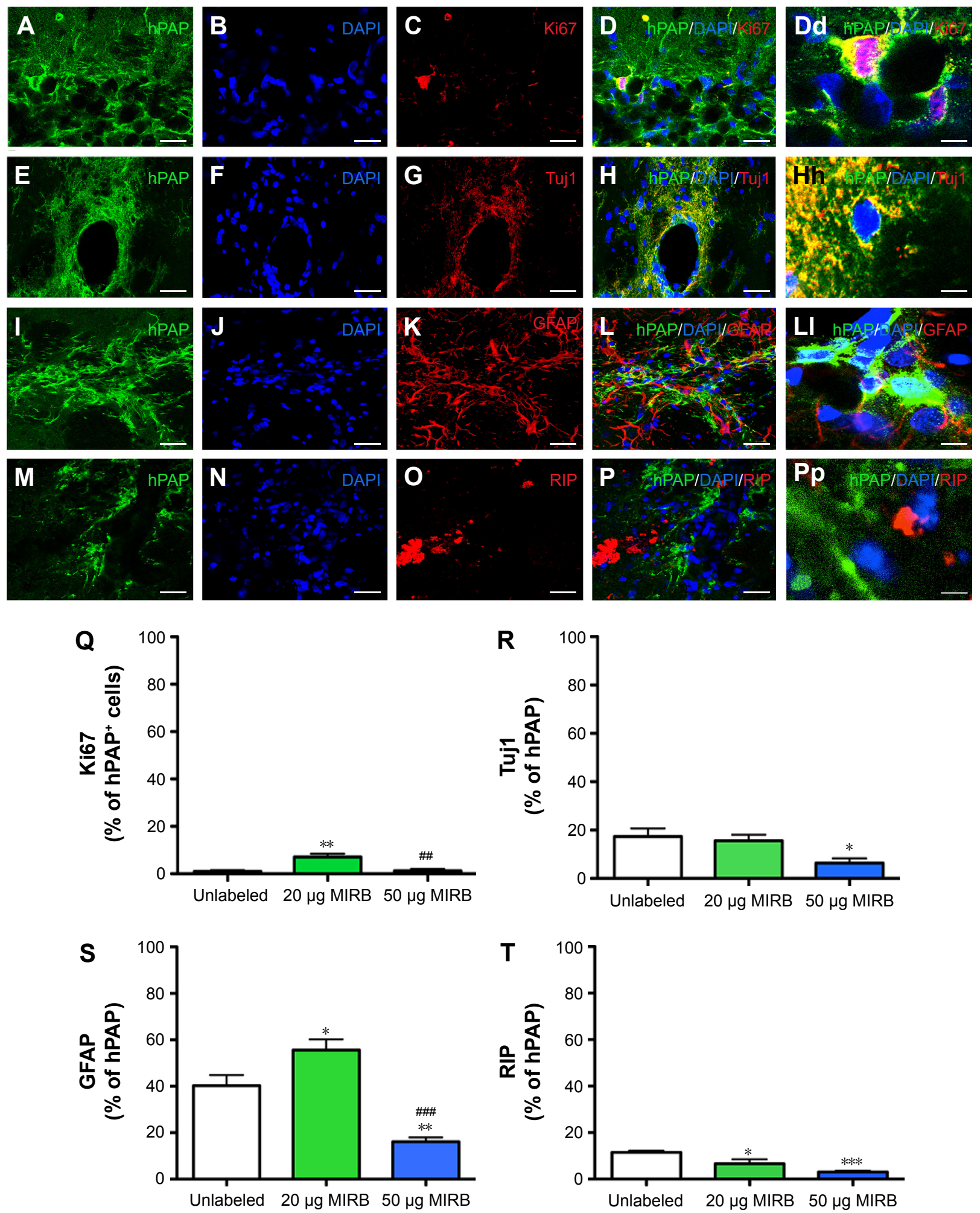

Figure $\mathbf{5}$ The proliferation and differentiation of transplanted NSCs are altered by MIRB labeling.

Notes: We examined proliferation in the hPAP NSC grafts in vivo via Ki67 expression ([A-D] and higher magnification image in [Dd]), show representative images from an animal grafted with unlabeled NSCs). The number of Ki67 cells within control grafts was negligible, with no changes seen in $50 \mu g$ grafts and increased numbers noted in the $20 \mu \mathrm{g}$ grafts $(\mathbf{Q})$. When differentiation was assessed through immunohistochemical staining with antibodies targeting Tuj I, GFAP, and RIP ([E-P] with corresponding higher magnification images in [Dd, $\mathrm{Hh}, \mathrm{LI}$, and Pp]), a severe reduction in the expression of all three antigens was noted within $50 \mu \mathrm{g}$ grafts compared to control (R-T). Interestingly, $20 \mu \mathrm{g}$ grafts showed increased GFAP but reduced RIP immunoreactivity, indicating that the glial differentiation of these cells had been affected. $* P<0.05$, $* * P<0.0$ I, $* * * P<0.00$ I compared to control, ${ }^{\# P}<0.0$ I, ${ }^{\# P}<0.00$ I compared to $20 \mu \mathrm{g}$, one-way ANOVA with post hoc Tukey's test. Scale bars: $30 \mu \mathrm{m}(\mathbf{A}-\mathbf{P})$ and I0 $\mu \mathrm{m}$ (Dd, Hh, Ll, and Pp).

Abbreviations: NSC, neural stem cell; MIRB, Molday ION Rhodamine B; ANOVA, analysis of variance. 
immunostaining with neuronal (Tuj1, Figure 5E-H; higher magnification image in $\mathrm{h}$ ) and glial (GFAP and RIP, Figure 5I-L and M-P; higher magnification images in Ll and Pp) antigens, it was determined that differentiation into neurons, astrocytes, and oligodendrocytes was significantly reduced within $50 \mu \mathrm{g}$ MIRB-labeled NSC grafts (Figure 5R, S, and T). In comparison to NSC grafts labeled with $20 \mu \mathrm{g}$ MIRB, astrocytic differentiation increased, oligodendrocyte differentiation decreased, and no changes were seen in neuronal differentiation. Overall, these data indicate that both concentrations of MIRB markedly altered the lineage differentiation pattern of the transplanted NSCs in vivo.

\section{MIRB promotes an exaggerated microglial response to NSC grafting}

CD68 (activated microglial marker) immunoreactivity surrounding the NSC grafts was examined at 1 month post-NSC transplantation to assess the immune response to grafting. In comparison to animals receiving unlabeled NSCs (Figure 6A-D), we observed that increased CD68+ microglia were present in animals transplanted with 20 and $50 \mu \mathrm{g}$ MIRB-labeled NSCs (Figure 6E-L). Quantitative analysis supported the qualitative impressions, and indicated that the total area of the striatum-inhabited $\mathrm{CD}^{+} 8^{+}$ cells, and the density of $\mathrm{CD} 68^{+}$cells within these areas, was significantly higher in animals injected with $50 \mu \mathrm{g}$ MIRBlabeled NSCs compared to animals receiving control NSCs (Figure 6R and $\mathrm{S}$ ). With respect to the animals receiving $20 \mu \mathrm{g}$ MIRB-labeled NSCs, the total area of the striatum occupied by the $\mathrm{CD}^{+} 8^{+}$cells was significantly higher, but the density of $\mathrm{CD}^{+} 8^{+}$microglia did not vary significantly compared to animals receiving control NSCs. In addition, interestingly, many $\mathrm{CD}^{+} 8^{+}$microglia located adjacent to $50 \mu \mathrm{g}$ MIRB-labeled NSCs displayed intracellular accumulation of MIRB as indicated by rhodamine fluorescence (Figure 6M-Q, arrows). This suggested that these activated microglial cells had possibly engulfed MIRB extruded by dying donor NSCs. Furthermore, a higher CD68 ${ }^{+}$cell presence was also observed in the $20 \mu \mathrm{g}$ MIRB group compared to control animals (Figure 6R, S, and E-H, arrow).

\section{Discussion}

The present study is novel in its comprehensive investigation of the influence of USPIO labeling on NSC biology, in parallel with dynamic MRI-based tracking of the NSCs, both in vitro and in vivo. Our results indicate that the USPIO MIRB can efficiently label NSCs and support their MR-based tracking in brain tissues; however, it affects the viability and regenerative capacity of the NSCs. In particular, although there were differences between the effects of the two doses of MIRB examined in the study, they both influenced the survival and proliferation, as well as differentiation rate and phenotype of NSCs in culture and after neural transplantation. Furthermore, the grafting of MIRB-labeled NSCs altered the host immune response, leading to an increased presence of activated $\mathrm{CD} 68^{+}$microglia in transplanted regions. Moreover, these activated host microglia appeared to have engulfed MIRB and/or MIRB-labeled NSCs, especially at the higher MIRB dose used, confounding the interpretation of the in vivo MRI signal dropout. These data advocate caution, and a systematic assessment of SPIObased NSC labeling agents before they are considered for clinical translation.

In vitro, it was determined that MIRB (both 20 and $50 \mu \mathrm{g}$ doses) was well internalized into the NSCs, although labeling was found to be more uniform and stronger with the $50 \mu \mathrm{g}$ dose. Also, the $50 \mu \mathrm{g}$ labeling promoted a better MR contrast signal in a single-cell suspension, indicating that this dose was probably better suited for the tracking of individual cells. Nevertheless, this particular dose of MIRB also significantly reduced the viability, proliferation, and differentiation (into neurons, astrocytes, and oligodendrocytes) of the NSCs. Interestingly, on the other hand, the $20 \mu \mathrm{g}$ dose did not affect the viability or regenerative ability of the NSCs, but it changed the phenotype of the differentiated cells. In particular, it promoted a more mature morphology of neurons and oligodendrocytes, and induced the production of predominantly fibrous astrocytes compared to the protoplasmic variety found in control cultures. Although the overall number of neuronal and glial cells generated in the $20 \mu \mathrm{g}$ cultures was similar to control cultures, the aforementioned change in their morphology could result in very different functional consequences in vitro and after in vivo grafting of these cells under both physiological and pathological scenarios. ${ }^{3,4,17,23}$ The exact nature of molecular and functional changes of NSCs induced by MIRB labeling will be investigated in future studies.

In vivo, grafted areas of the striatum containing both 20 and $50 \mu \mathrm{g}$ MIRB-labeled NSCs could be identified via an MRI signal dropout in the short term (3 and 7 days post-transplantation) as well as the long term (1 month post-transplantation). This signal dropout did not appreciably change in size, shape, or location over the 28 days studied. However, similar to what was observed in vitro, the $50 \mu \mathrm{g}$-labeled NSC grafts exhibited reduced survival, and a significantly decreased ability to generate all the three major neural lineages upon differentiation also in vivo. In contrast, proliferation was significantly amplified within 

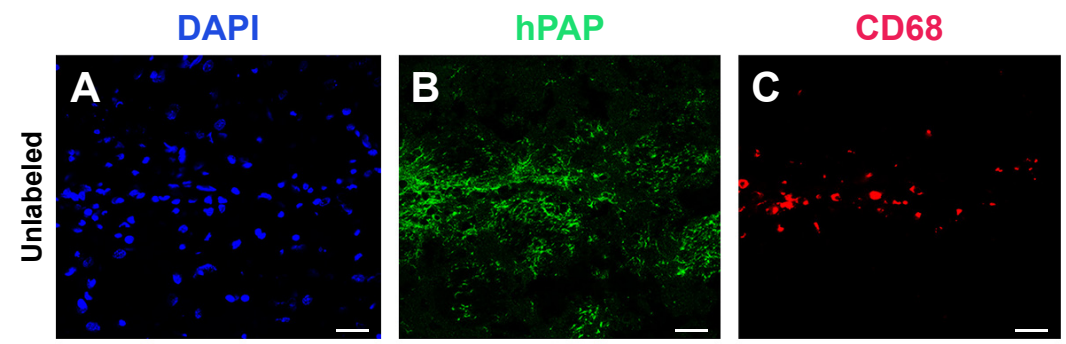

\section{DAPI/hPAP/CD68}
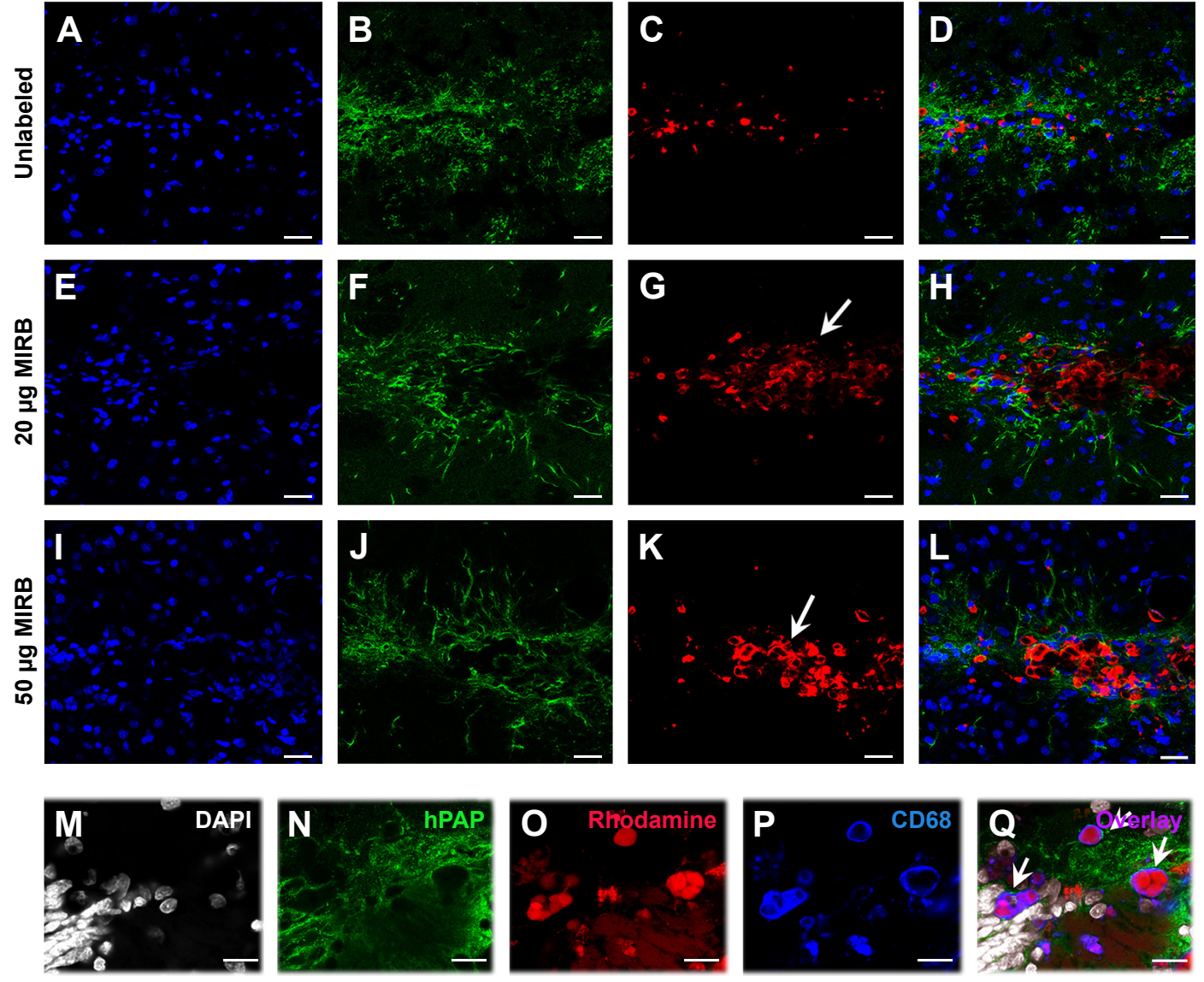

$\mathbf{R}$
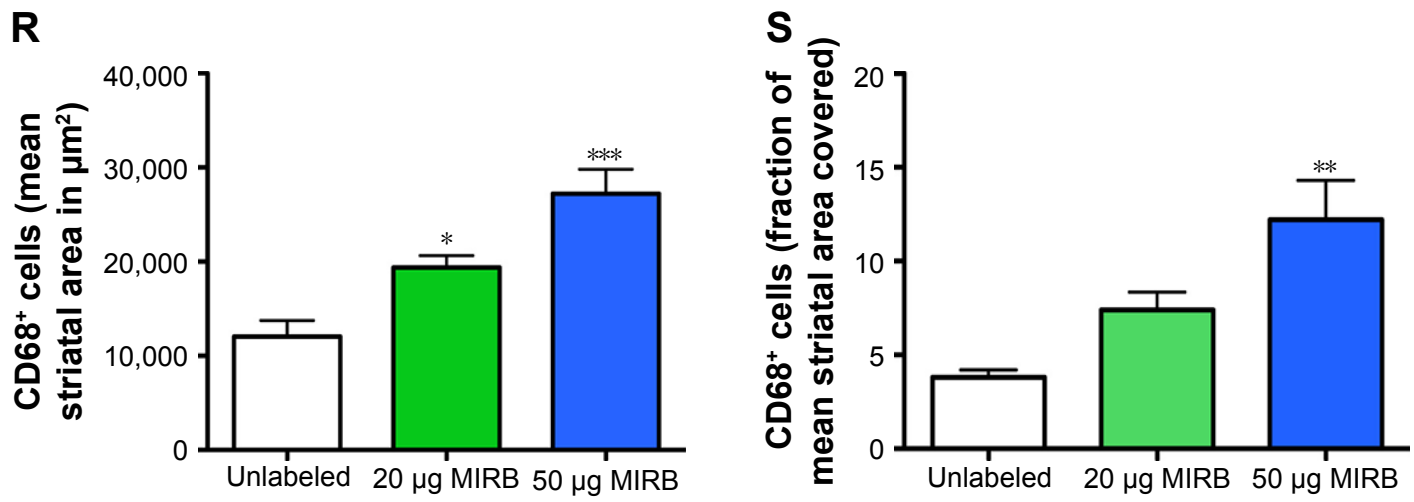

Figure 6 Transplantation of MIRB-labeled NSCs induces increased microglial activation and ectopic labeling of CD68 microglia.

Notes: We immunostained brain sections obtained from all animal groups with antibodies targeting the activated microglial marker, CD68. It was observed that there were greater numbers of $\mathrm{CD}_{6} 8^{+}$microglia (red) in the striata of animals receiving both 20 (E-H, arrow) and $50 \mu \mathrm{g}$ (I-L, arrow) MIRB-labeled NSCs, compared to those receiving control unlabeled NSCs (A-D). This qualitative impression was confirmed via quantitative methods which determined that the total striatal area covered with CD68 ${ }^{+}$cells, as well as the density of CD68+ cells, was increased in animals with MIRB-labeled NSC grafts, although this was significantly higher in the $50 \mu g$ case (R and $\mathbf{S})$. Furthermore, a number of $\mathrm{CD} 68^{+}$microglia (P) present adjacent to the hPAP NSC grafts were noted to contain MIRB (O) suggesting the engulfment of extravasated MIRB by these phagocytic cells. Arrows in the overlay image in (Q) point to CD68 cells which contain engulfed MIRB. $* P<0.05$, $* * P<0.0$ I, $* * * P<0.00$ I, one-way ANOVA with post hoc Tukey's test. Scale bars: $30 \mu \mathrm{m}(\mathbf{A}-\mathbf{L})$ and $20 \mu \mathrm{m}(\mathbf{M}-\mathbf{Q})$.

Abbreviations: MIRB, Molday ION Rhodamine B; NSC, neural stem cell; ANOVA, analysis of variance; DAPI, 4',6-diamidino-2-phenylindole.

the $20 \mu \mathrm{g}$ grafts (there were a higher number of Ki67 cells), and the glial differentiation was altered (greater expression of astrocyte antigen GFAP but reduced expression of the oligodendrocyte antigen RIP). Interestingly, although such significant changes in proliferation and glial differentiation had not been observed from $20 \mu \mathrm{g}$ MIRB labeling in vitro, in support of the in vivo data, there was a trend toward increased Ki67 and GFAP cells, and reduced RIP cells, in culture (Figures 2N and 3P).

In vivo, a signal dropout was also observed in rats transplanted with unlabeled cells and in sham rats that received injection of buffer only (Figure S2). While the signal 
dropout persisted over the course of the experiment, it was not circular in shape like that of the labeled cells and changed appearance over time. The signal dropout without labeling is likely due to blood products, such as hemosiderin, which are caused by the injection and are known to persist for months after injection. ${ }^{22,24,25}$ Persistent signal dropout after needle transplantation of labeled and unlabeled cells is consistent with previous reports and remains a confounding factor for negative-contrast MRI cell tracking. Positive-contrast MRI experiments with iron oxide-labeled cells have been developed and are being investigated to address this problem but are not yet in common use. , 26,27 $^{8}$

There were also increased numbers of activated microglia existing in regions transplanted with the MIRB-labeled NSCs. This phenomenon was substantially more pronounced in the $50 \mu \mathrm{g}$ case, compared to animals receiving $20 \mu \mathrm{g}$ MIRB-labeled NSCs, and can be attributed to the observed reduced survival of the $50 \mu \mathrm{g}$ grafts which led to the release of MIRB from dying cells. In addition, given that active microglia can substantially reduce the viability of grafted cells and alter their function through the expression of specific proinflammatory cytokines and other toxic species, ${ }^{28}$ the reduced size of the $50 \mu \mathrm{g}$ grafts in our study could in part be attributed to such a secondary effect. This concept is supported by the increased presence of microglia surrounding even the $20 \mu \mathrm{g}$ MIRB-labeled grafts (where no significant cell death/loss was noted), suggesting that MIRB may also be directly affecting the intrinsic biology of NSCs, in particularly how these cells interacted with host immune cells.

In addition, it is plausible that MIRB could also have directly affected NSC viability by increasing intracellular oxidative stress. ${ }^{29,30}$ In this regard, it is well established that iron can react with oxidant molecules, via classic Fenton reactions, further amplifying the production of damaging ROS that cause oxidative stress. ${ }^{31}$ It is also known that SPIOs are capable of promoting the generation of intracellular ROS, resulting in oxidative stress and cellular toxicity. ${ }^{32,33}$ Also, our in vitro analysis showed high ROS production in the NSCs after MIRB labeling using the $50 \mu \mathrm{g}$ dose, suggesting that the ROS production probably contributed to the reduced survival and regeneration of the NSCs. Interestingly, some ROS generation was also noted in the $20 \mu \mathrm{g}$-treated NSCs. Here, no obvious associated cytotoxic effects were observed, although increases in proliferative capacity and glial differentiation were seen. Given that the intracellular redox state (whether oxidized or reduced) plays a crucial role in determining NSC regenerative function, we suggest that the production of ROS (and resulting alteration in intracellular redox) may have altered the proliferation and morphological differentiation of the $20 \mu \mathrm{g} / \mathrm{mL}$ MIRB-labeled NSCs. ${ }^{34}$ Lastly, given that microglia are capable of producing factors that can modulate NSC regeneration, such a mechanism could have further supported the altered proliferation and differentiation observed within the MIRB-labeled grafts. ${ }^{31,35}$

Most strikingly, CD68 microglia expressing rhodamine and containing multiple nuclei were noticed adjacent to MIRB grafts, especially in the $50 \mu \mathrm{g}$ case, suggesting that these activated immune cells had probably engulfed MIRB/ MIRB-labeled NSCs. This presents a serious problem, since such MIRB-labeled microglial cells could contribute to the MRI signal dropout and produce a false-positive result. ${ }^{13}$ In fact, this probably occurred in animals transplanted with the $50 \mu \mathrm{g}$ MIRB-labeled NSCs, where although the MRI signal dropout was strongest, the graft size was determined to be smaller on average compared to animals receiving $20 \mu \mathrm{g}$ (or unlabeled) NSC grafts (explained in the "Detection and assessment of viability and migration of transplanted MIRBlabeled NSCs via extended MRI-based in vivo tracking and post hoc histology" section).

In contrast to our work, Shen et al have conducted an analysis of human NSCs and observed no significant changes in viability, proliferation, and differentiation, after MIRB labeling. ${ }^{10,16,36}$ However, these studies used a different dose of MIRB ( $20 \mu \mathrm{g} / \mathrm{mL}$ dose for 48-72 hours), focused on shortterm effects of MIRB, and did not include an investigation of the multipotentiality or morphology of the NSCs upon differentiation, which could have accounted for the differences. Other studies from Guzman et al and Kallur et al have also examined NSC biology after SPIO labeling. ${ }^{37,38}$ Here, human NSCs were labeled with the iron oxide agents Ferridex or Endorem, and their in vitro viability, proliferation, and differentiation into Tuj $1^{+}$neurons and $\mathrm{GFAP}^{+}$glia were examined. Additionally, the long-term survival and migration of SPIO-labeled donor NSCs after transplantation and the differentiation of the grafted cells were also assessed. Broadly, data from these studies revealed no significant differences between the viability, fate, and migratory capacity of unlabeled NSCs and those labeled with SPIO particles. However, Kallur et al did observe a reduction in proliferation (through Ki67 staining), and an altered neuronal differentiation rate (Tuj $1^{+}$and Doublecortin ${ }^{+}$cells), in a sect of the SPIO-labeled NSC population. Nevertheless, these studies lacked a detailed in vitro and in vivo analysis of NSC morphology, behavior, and fate, as well as the immune response related to grafting. On the other hand, in support of our current findings, it has been reported that SPIOs can 
reduce NSC motility, be exocytosed by live NSCs, and also be internalized by surrounding microglia when there is grafted cell death to complicate the interpretation of the in vivo MR contrast signal. ${ }^{39,40}$

\section{Conclusion}

This study indicates that USPIO agents can affect the viability and regenerative capacity of NSCs, even at seemingly low nontoxic concentrations, and alter graft-host immune dynamics after NSC transplantation into the adult nervous system. The specific nature and the optimal functioning of grafted cells are of essence in determining the success of any cell therapeutic approach. Data from our investigation strongly suggest that a further examination of USPIO agents might be needed before they can safely and effectively be used for clinical translation.

\section{Acknowledgments}

We thank Kayla Yu and Quentin Remley for their technical assistance on the histological aspects of the project. We are also grateful to Doug Cromey (Arizona Cancer Center Imaging Core Cancer Center Support Grant [P30 CA023074]) for his technical support with confocal microscopy and image analysis. This work was supported by intramural funds from The University of Arizona to LM.

\section{Author contributions}

AU, SR, and MJC contributed to experimental design, collection and assembly of data, data analysis and interpretation, and manuscript writing. MV contributed to experimental design, collection and assembly of data, data analysis and interpretation, and manuscript revision. EY contributed to collection and assembly of data, data analysis and interpretation, and manuscript revision. TT contributed to conception and design, collection and assembly of data, data analysis and interpretation, and manuscript revision. LM contributed to conception and design, collection and assembly of data, data analysis and interpretation, manuscript writing and revision, financial support, and final approval of manuscript.

\section{Disclosure}

The authors report no conflicts of interest in this work.

\section{References}

1. Breunig JJ, Haydar TF, Rakic P. Neural stem cells: historical perspective and future prospects. Neuron. 2011;70(4):614-625.

2. De Feo D, Merlini A, Laterza C, Martino G. Neural stem cell transplantation in central nervous system disorders: from cell replacement to neuroprotection. Curr Opin Neurol. 2012;25(3):322-333.
3. Madhavan L, Collier TJ. A synergistic approach for neural repair: cell transplantation and induction of endogenous precursor cell activity. Neuropharmacology. 2010;58(6):835-844.

4. Madhavan L, Daley BF, Davidson BL, et al. Sonic hedgehog controls the phenotypic fate and therapeutic efficacy of grafted neural precursor cells in a model of nigrostriatal neurodegeneration. PLoS One. 2015; 10(9):e0137136.

5. Madhavan L, Daley BF, Paumier KL, Collier TJ. Transplantation of subventricular zone neural precursors induces an endogenous precursor cell response in a rat model of Parkinson's disease. J Comp Neurol. 2009;515(1):102-115.

6. Mazzini L, Gelati M, Profico DC, et al. Human neural stem cell transplantation in ALS: initial results from a phase I trial. J Transl Med. 2015;13:17.

7. Feldman EL, Boulis NM, Hur J, et al. Intraspinal neural stem cell transplantation in amyotrophic lateral sclerosis: phase 1 trial outcomes. Ann Neurol. 2014;75(3):363-373.

8. Korchinski DJ, Taha M, Yang R, Nathoo N, Dunn JF. Iron oxide as an MRI contrast agent for cell tracking. Magn Reson Insights. 2015; 8(Suppl 1):15-29.

9. Chen CC, Ku MC, Jayaseema DM, Lai JS, Hueng DY, Chang C. Simple SPION incubation as an efficient intracellular labeling method for tracking neural progenitor cells using MRI. PLoS One. 2013;8(2): e56125.

10. Shen WB, Plachez C, Chan A, et al. Human neural progenitor cells retain viability, phenotype, proliferation, and lineage differentiation when labeled with a novel iron oxide nanoparticle, Molday ION Rhodamine B. Int J Nanomedicine. 2013;8:4593-4600.

11. Aswendt M, Henn N, Michalk S, et al. Novel bimodal iron oxide particles for efficient tracking of human neural stem cells in vivo. Nanomedicine (London). 2015;10(16):2499-2512.

12. Kim SJ, Lewis B, Steiner MS, Bissa UV, Dose C, Frank JA. Superparamagnetic iron oxide nanoparticles for direct labeling of stem cells and in vivo MRI tracking. Contrast Media Mol Imaging. 2016;11(1): 55-64.

13. Ramos-Gomez M, Seiz EG, Martinez-Serrano A. Optimization of the magnetic labeling of human neural stem cells and MRI visualization in the hemiparkinsonian rat brain. J Nanobiotechnology. 2015;13:20.

14. Addicott B, Willman M, Rodriguez J, et al. Mesenchymal stem cell labeling and in vitro MR characterization at $1.5 \mathrm{~T}$ of new SPIO contrast agent: Molday ION Rhodamine-B. Contrast Media Mol Imaging. 2011;6(1):7-18.

15. Zhang G, Na Z, Ren B, Zhao X, Liu W. Impacts of fluorescent superparamagnetic iron oxide (SPIO)-labeled materials on biological characteristics and osteogenesis of bone marrow mesenchymal stem cells (BMSCs). Int J Clin Exp Med. 2015;8(8):12172-12181.

16. Shen WB, Plachez C, Tsymbalyuk O, et al. Cell-based therapy in TBI: Magnetic retention of neural stem cells in vivo. Cell Transplant. Epub 2015 Sep 21.

17. Madhavan L, Daley BF, Sortwell CE, Collier TJ. Endogenous neural precursors influence grafted neural stem cells and contribute to neuroprotection in the parkinsonian rat. Eur J Neurosci. 2012;35(6): 883-895.

18. Han SS, Kang DY, Mujtaba T, Rao MS, Fischer I. Grafted lineagerestricted precursors differentiate exclusively into neurons in the adult spinal cord. Exp Neurol. 2002;177(2):360-375.

19. Koh SH, Noh MY, Cho GW, Kim KS, Kim SH. Erythropoietin increases the motility of human bone marrow-multipotent stromal cells (hBM-MSCs) and enhances the production of neurotrophic factors from hBM-MSCs. Stem Cells Dev. 2009;18(3):411-421.

20. Chen M, Tian S, Yang X, Lane AP, Reed RR, Liu H. Wnt-responsive $\mathrm{Lgr}^{+}$globose basal cells function as multipotent olfactory epithelium progenitor cells. J Neurosci. 2014;34(24):8268-8276.

21. Nan H, Huang J, Li H, Li Q, Liu D. Assessment of biological characteristics of adipose tissue-derived stem cells co-labeled with Molday ION Rhodamine $\mathrm{B}^{\mathrm{TM}}$ and green fluorescent protein in vitro. $\mathrm{Mol} \mathrm{Med}$ Rep. 2013;8(5):1446-1452. 
22. Williams SJ. Development and Appraisal of MRI Contrast Agents for the In Vivo Analysis of Stem Cell Grafts [PhD thesis]. Cardiff, Wales, UK: Cardiff University; 2012. Available from: http://orca.cf.ac.uk/43157/. Accessed April 13, 2016.

23. Ourednik V, Ourednik J. Graft/host relationships in the developing and regenerating CNS of mammals. Ann N Y Acad Sci. 2005; 1049:172-184.

24. Schommer K, Kallenberg K, Lutz K, Bartsch P, Knauth M. Hemosiderin deposition in the brain as footprint of high-altitude cerebral edema. Neurology. 2013;81(20):1776-1779.

25. Atlas SW, editor. Head trauma. In: Magnetic Resonance Imaging of the Brain and Spine. Philadelphia, PA, USA: Lippincott Williams \& Wilkins; 2009.

26. Han SH, Cho JH, Jung HS, et al. Robust MR assessment of cerebral blood volume and mean vessel size using SPION-enhanced ultrashort echo acquisition. NeuroImage. 2015;112:382-389.

27. Scharlach C, Warmuth C, Schellenberger E. Determination of blood circulation times of superparamagnetic iron oxide nanoparticles by $\mathrm{T}^{*} *$ relaxometry using ultrashort echo time (UTE) MRI. Magn Reson Imaging. 2015;33(9):1173-1177.

28. Kaminska B, Mota M, Pizzi M. Signal transduction and epigenetic mechanisms in the control of microglia activation during neuroinflammation. Biochim Biophys Acta. 2016;1862(3):339-351.

29. Stroh A, Boltze J, Sieland K, et al. Impact of magnetic labeling on human and mouse stem cells and their long-term magnetic resonance tracking in a rat model of Parkinson disease. Mol Imaging. 2009;8(3): 166-178.

30. Petters C, Thiel K, Dringen R. Lysosomal iron liberation is responsible for the vulnerability of brain microglial cells to iron oxide nanoparticles: comparison with neurons and astrocytes. Nanotoxicology. 2016; $10(3): 332-342$.

31. Valko M, Jomova K, Rhodes CJ, Kuca K, Musilek K. Redox- and nonredox-metal-induced formation of free radicals and their role in human disease. Arch Toxicol. 2016;90(1):1-37.
32. Imam SZ, Lantz-McPeak SM, Cuevas E, et al. Iron oxide nanoparticles induce dopaminergic damage: in vitro pathways and in vivo imaging reveals mechanism of neuronal damage. Mol Neurobio. 2015; 52(2):913-926.

33. Luo C, Li Y, Yang L, Wang X, Long J, Liu J. Superparamagnetic iron oxide nanoparticles exacerbate the risks of reactive oxygen speciesmediated external stresses. Arch Toxicol. 2015;89(3):357-369.

34. Madhavan L. Redox-based regulation of neural stem cell function and Nrf2. Biochem Soc Trans. 2015;43(4):627-631.

35. Sato K. Effects of microglia on neurogenesis. Glia. 2015;63(8): 1394-1405.

36. Shen WB, Vaccaro DE, Fishman PS, Groman EV, Yarowsky P. SIRB, sans iron oxide rhodamine B, a novel cross-linked dextran nanoparticle, labels human neuroprogenitor and SH-SY5Y neuroblastoma cells and serves as a USPIO cell labeling control. Contrast Media Mol Imaging. Epub 2016 Jan 25.

37. Guzman R, Uchida N, Bliss TM, et al. Long-term monitoring of transplanted human neural stem cells in developmental and pathological contexts with MRI. Proc Nat Acad Sci U S A. 2007; 104(24):10211-10216.

38. Kallur T, Farr TD, Bohm-Sturm P, Kokaia Z, Hoehn M. Spatiotemporal dynamics, differentiation and viability of human neural stem cells after implantation into neonatal rat brain. Eur J Neurosci. 2011;34(3):382-393.

39. Berman SC, Galpoththawela C, Gilad AA, Bulte JW, Walczak P. Longterm MR cell tracking of neural stem cells grafted in immunocompetent versus immunodeficient mice reveals distinct differences in contrast between live and dead cells. Magn Reson Med. 2011;65(2):564-574.

40. Cromer Berman SM, Kshitiz, Wang CJ, et al. Cell motility of neural stem cells is reduced after SPIO-labeling, which is mitigated after exocytosis. Magn Reson Med. 2013;69(1):255-262. 


\section{Supplementary materials}
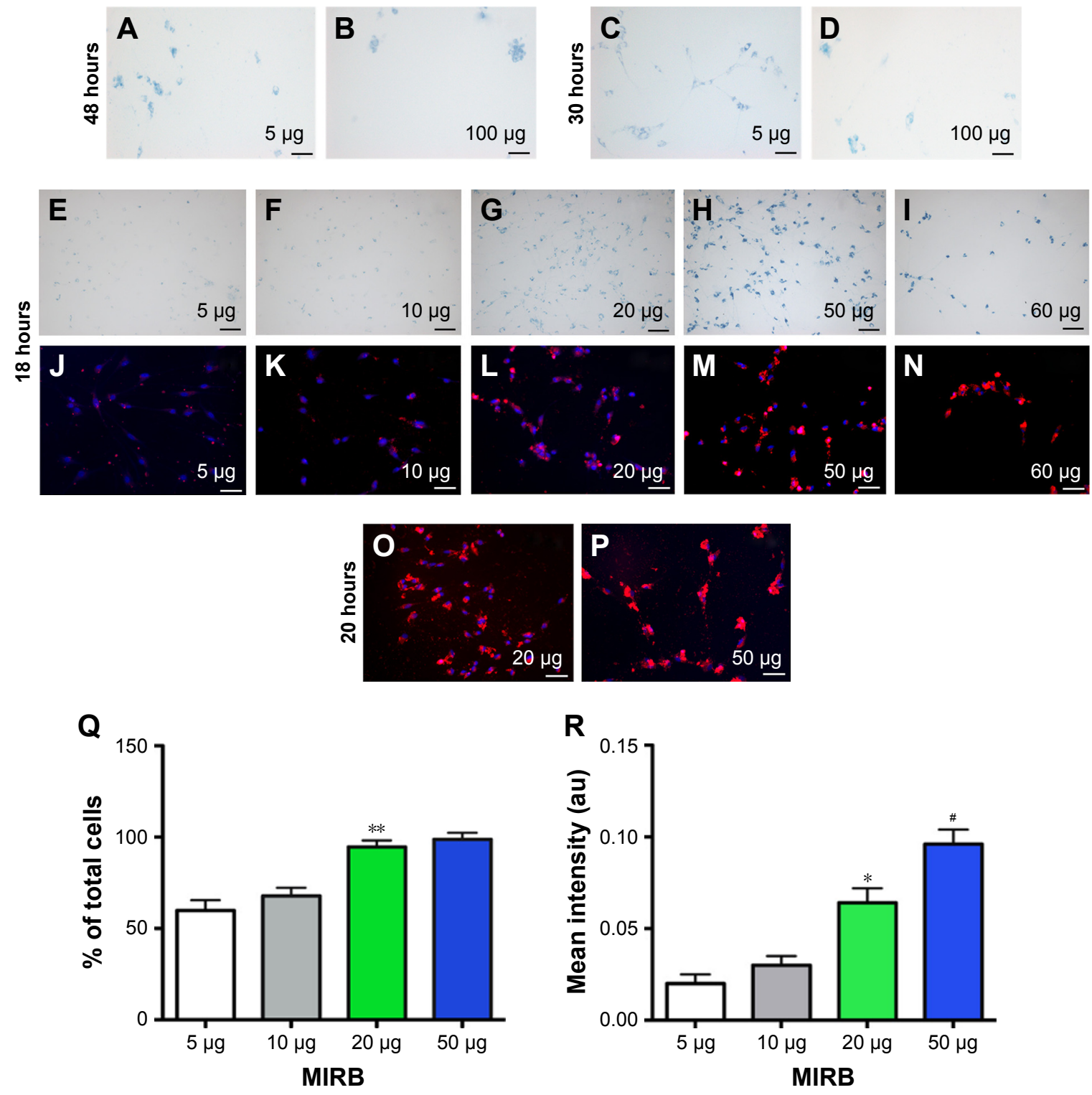

Figure SI Optimization of MIRB dose.

Notes: Based on studies regarding MIRB labeling of NSCs reported in literature, NSCs were treated first with 5, 10, 20, 50, 60, 80, or I00 $\mu \mathrm{g}$ Fe/mL of MIRB for a time period of 30 or 48 hours (A-D). Here, an obvious reduction in cell viability was noted even at the $5 \mu \mathrm{g}$ dose (A and $\mathbf{C})$. Therefore, we reduced the labeling time to I 8 hours, again after reviewing the literature. Under these conditions, cells labeled with $5,10,20,50 \mu g$ doses did not qualitatively show a significant reduction in cell viability (E-H and $\mathbf{J}-\mathbf{M})$. As shown, a loss of cells was already seen at the $60 \mu \mathrm{g}$ level (I and $\mathbf{N}$ ). The 20, 50, and $60 \mu \mathrm{g}$ doses showed good labeling, as confirmed via the Prussian blue staining (G-I) and the detection of rhodamine autofluorescence $(\mathbf{L}-\mathbf{N})$. We further increased the incubation period to 20 hours to refine the labeling in cells treated with 20 and $50 \mu g / m L$ MIRB ( $\mathbf{O}$ and $\mathbf{P}$ ). The labeling efficiency (percentage of labeled cells) as well as intensity (quantitation of rhodamine fluorescence), after 20 hours of MIRB labeling, in doses up to $50 \mu \mathrm{g}$, is shown in $(\mathbf{Q}$ and $\mathbf{R})$. ${ }^{* P}<0.05$ compared to $10 \mu \mathrm{g}$, ${ }^{*} P<0.05$ compared to $20 \mu \mathrm{g},{ }^{*} * P<0.00$ I compared to $10 \mu \mathrm{g}$, one-way ANOVA. Scale bars: $20 \mu \mathrm{m}$. Abbreviations: MIRB, Molday ION Rhodamine B; NSCs, neural stem cells; ANOVA, analysis of variance. 

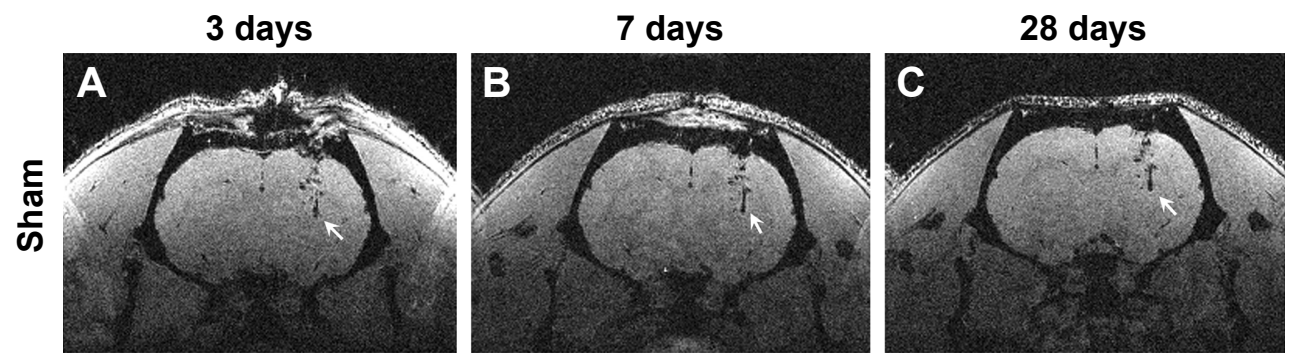

Figure S2 MRI of sham animals.

Notes: Animals injected with buffer, as opposed to cells, were also scanned using 3D gradient-echo MRI. The imaging protocol and parameters were identical to those mentioned in the main paper. As reported in the literature, at all the three time points ( 3,7 , and 28 days post-transplantation) assessed, signal dropout was seen corresponding to the needle track area (arrows in [A-C]).

Abbreviation: MRI, magnetic resonance imaging.

\section{Publish your work in this journal}

The International Journal of Nanomedicine is an international, peerreviewed journal focusing on the application of nanotechnology in diagnostics, therapeutics, and drug delivery systems throughout the biomedical field. This journal is indexed on PubMed Central, MedLine, CAS, SciSearch $®$, Current Contents ${ }^{\circledR} /$ Clinical Medicine,
Journal Citation Reports/Science Edition, EMBase, Scopus and the Elsevier Bibliographic databases. The manuscript management system is completely online and includes a very quick and fair peer-review system, which is all easy to use. Visit http://www.dovepress.com/ testimonials.php to read real quotes from published authors. 